\title{
Una Plataforma Web para Gestionar los Derechos de Propiedad Intelectual Resultantes de la Investigación Universitaria
}

\author{
Diego Acuña', Rodolfo Schmal', Patricia Klein ${ }^{3}$
}

\begin{abstract}
The paper show the development of a business process model and a web site for manage the industrial property rights linked to results of programs and projects executed in Talca's University (Chile). The work begin with an exploratory research followed by the construction of a business process model using BPMN Business Process Modeling Notation). Finally was developed a web site using XP (eXtreme Programming) methodology of rapid project development.

Results obtained -business process model and web site-, make possible formalize process, rules definition, and the control of applications associated to industrial property rights.
\end{abstract}

Keywords: Web site; industrial property rights; university research; business process model; management.

\section{Resumen}

Se presenta el desarrollo de un modelo de procesos y una plataforma web para gestionar los derechos de propiedad industrial asociados a los resultados de los programas y proyectos de investigación que se ejecutan en la Universidad de Talca (Chile). El trabajo se inició con una investigación exploratoria seguida de la construcción de un modelo de procesos haciendo uso de la notación BPMN (Business Process Modeling Notation). Finalmente se procedió a desarrollar la plataforma web aplicando la metodología de desarrollo ágil de proyectos XP (eXtreme Programming). El resultado alcanzado -el modelo de procesos y la plataforma web-, han posibilitado la formalización de procesos, la definición de reglas, la identificación de roles por parte de los distintos actores involucrados, y están facilitando el monitoreo de las solicitudes de protección de los derechos de propiedad industrial en la Universidad.

Palabras claves: Plataforma web; derechos de propiedad industrial; investigación universitaria; modelo de procesos; gestión.

\footnotetext{
' Escuela Ing. Informática Empresarial, Universidad de Talca, Avda.Lircay s/n, Talca-Chile, fono: 7I-200354, Email: dacuna@alumnos.utalca.cl

2 Facultad de Ciencias Empresariales, Universidad de Talca, Avda.Lircay s/n, Talca-Chile, fono: 7I-200354, Email: rschmal@utalca.cl

${ }^{3}$ Unidad de Patentamiento, Universidad de Talca, Avda.Lircay s/n, Talca-Chile, fono: 7I-200354, Email: pklein@utalca.cl
} 


\section{Introducción}

Con mayor o menor énfasis, la razón de ser de las universidades ha estado asociada a la generación (investigación), transmisión (docencia) y difusión (extensión) del conocimiento. Sin embargo, en las últimas décadas ha surgido un nuevo rol para las universidades en su relación con la sociedad, el de la comercialización de los resultados de la investigación científico-tecnológica que se desarrolla en su interior. La creciente relevancia que ha ido adquiriendo el conocimiento científico para el desarrollo de nuevos negocios, le han asignado a las universidades un significativo rol en el ámbito de la innovación. Por otra parte, los crecientes costos que deben afrontar las universidades y las dificultades para proveerse de fondos públicos para financiar estos costos, explican el interés por la comercialización de los resultados de una investigación empujada más por requerimientos de mercado que por la curiosidad científica (Gibbons, 2004).

Este interés se enmarca dentro de un esquema de mayor competitividad y liberalización a nivel mundial, lo que en términos económicos se ha traducido en la proliferación de tratados de libre comercio que incluyen cláusulas destinadas al fortalecimiento de los derechos de propiedad intelectual (DPI), concibiéndose su existencia como una condición para la suscripción de acuerdos y/o contratos. Por otra parte, las universidades, particularmente las públicas, cuyo financiamiento solía ser fijo y estable, está dejando de serlo, tendiendo a incluirse un componente de carácter concursable (Brunner, 2009). Crecientemente, estos fondos concursables están siendo destinados a inducir el desarrollo de proyectos cooperativos entre universidades y empresas, y en los que se alienta la generación de patentes. Es así como los DPI se visualizan como mecanismos de protección y estímulo para la transferencia tecnológica desde las universidades hacia las empresas.

En USA, a partir de la ley Bayh Dole en 1980, se desató un fuerte proceso de comercialización de los resultados de la investigación en las universidades, con preeminencia de la investigación aplicada por sobre la básica, con consecuencias en el volumen de patentes generadas y en el número y valor de los contratos formalizados (Sampat et al., 2003; Movary et al., 200I). Según la estadística más reciente del 2008, el número de solicitudes $\mathrm{PCT}^{\prime}$ hechas por universidades asciende a casi dos mil unidades, lo que representa del orden del $1 \%$ del total de solicitudes de patentes requeridas a través del sistema PCT. De las solicitudes efectuadas por las universidades, destacan las universidades de Estados Unidos, en particular las del sistema universitario público de California, que concentran sobre el $20 \%$ del total. De las 15 universidades con mayor número de solicitudes, solo dos universidades no son de Estados Unidades: la Universidad de Tokio y la Universidad Nacional de Seúl (OMPI, 2009). Aunque los datos citados se refieren exclusivamente a las solicitudes de patente mediante los mecanismos del PCT, son elocuentes respecto del predominio de las universidades estadounidenses.

En las universidades de América Latina, y en Chile en particular, existe un fuerte rezago en estas materias (Zaldívar y OConnor, 2010; Plácido y Vitale, 2009; Schmal et al., 2006). En la Tabla I se presenta la evolución de las solicitudes de patentes por parte de las universidades chilenas en las últimas décadas, observándose que la última década concentra casi el $80 \%$ de las solicitudes, señal del creciente interés en proteger los DPI de los resultados de la investigación que se realiza en sus laboratorios. De hecho, la mayoría de las universidades aún no cuentan con políticas formales de protección a la propiedad intelectual, existiendo a lo más políticas informales en torno a los derechos de autor. Esta realidad se explica fundamentalmente porque la universidad ha sido visualizada como una institución diseminadora, antes que comercializadora de conocimientos. Esta nueva visión se debe al predominio actual de un pensamiento promercado.

\footnotetext{
' El Tratado de Cooperación en materia de Patentes (PCT, por sus siglas en inglés: Patent Cooperation Treaty) es un instrumento suscrito por más de 125 países en 1970 que instituyó un procedimiento unificado, de validez internacional, para la solicitud de patentes. Si bien este procedimiento de registro a través del PCT no sustituye el procedimiento formal de registro en cada país, sí facilita la homologación del trámite inicial de solicitud, particularmente cuando se busca la obtención de una patente en múltiples países.
} 


\begin{tabular}{|c|c|c|c|c|c|c|}
\hline & $\mathbf{1 9 6 1 - 1 9 7 0}$ & $\mathbf{1 9 7 1 - 1 9 8 0}$ & $\mathbf{1 9 8 1 - 1 9 9 0}$ & $\mathbf{1 9 9 1 - 2 0 0 0}$ & $\mathbf{2 0 0 1 - 2 0 1 0}$ & TOTAL \\
\hline \multirow{2}{*}{ Patentes } & 1 & 5 & 17 & 58 & 293 & 374 \\
\hline$\%$ & 0,3 & 1,3 & 4,5 & 15,5 & 78,3 & $100 \%$ \\
\hline
\end{tabular}

Tabla I: Evolución de las solicitudes de patentes por parte de las universidades chilenas del $\mathrm{CRUCH}^{2} /$ Fuente: Elaboración propia a partir de datos disponibles en INAPI (http://ion.inapi.cl)

En el anexo se puede observar que el $80 \%$ de las 374 solicitudes de patentes han sido presentados por 6 universidades, destacándose la Universidad de Concepción ${ }^{3}$ con más de 100 patentes solicitadas, representando más del $25 \%$ del total.
Junto con ser la universidad con mayor número de solicitudes de patentes, la Universidad de Concepción es también la que posee la mayor tasa de éxito en término del porcentaje de solicitudes de patentes concedidas (36\%) muy por encima de las restantes universidades. En cuanto a la evolución que han experimentado en el tiempo las patentes solicitadas y concedidas, la Tabla 2 muestra el crecimiento que han registrado en las últimas dos décadas, tanto las patentes solicitadas como las concedidas.

\begin{tabular}{|l|c|c|c|c|c|}
\hline Patentes & $\mathbf{1 9 7 1 - 1 9 8 0}$ & $\mathbf{1 9 8 1 - 1 9 9 0}$ & $\mathbf{1 9 9 1 - 2 0 0 0}$ & $\mathbf{2 0 0 1 - 2 0 1 0}$ & TOTAL \\
\hline Solicitadas & 2 & 5 & 29 & 65 & 101 \\
\hline Concedidas & 2 & 5 & 15 & 14 & 36 \\
\hline
\end{tabular}

Tabla 2. Evolución de patentes solicitadas y concedidas en la Universidad de Concepción./ Fuente: Elaboración propia a partir de datos disponibles en INAPI (http://ion.inapi.cl)

' En Chile, al año 2010 existe un total de 60 universidades, 16 estatales, 9 privadas tradicionales y 35 privadas, estas últimas sin aporte fiscal directo. Las 25 universidades estatales y privadas tradicionales constituyen el Consejo de Rectores de Universidades Chilenas (CRUCH). ${ }^{2}$ Universidad localizada en la Región del Bío Bío, al sur de Chile, a 500 km de Santiago de Chile, de carácter privada perteneciente al $\mathrm{CRUCH}$ y caracterizada por su fuerte vinculación con el medio en el que se inserta. 


\section{La Gestión de los DPI en las universidades}

La gestión de los DPI en las universidades ha estado adquiriendo relevancia como consecuencia del interés existente desde organizaciones nacionales (Instituto Nacional de Propiedad Industrial en el caso chileno) e internacionales (Organización Mundial de Propiedad Intelectual) por fomentar una cultura que aliente a creadores e innovadores a obtener y utilizar los derechos y activos de propiedad intelectual y generar conciencia de la necesidad de respetar dichos derechos. Es así como en su condición de polo de desarrollo tecnológico se ha estado fomentando la necesidad de que las universidades impulsen y desarrollen nuevas tecnologías, difundan su investigación, y desplieguen acciones destinadas a obtener fondos para apoyar el desarrollo de la investigación.

Surge entonces la opción de que las universidades implementen unidades organizacionales orientadas a facilitar la gestión de la propiedad intelectual en su seno, que además permitan potenciar los vínculos entre el sector académico y el sector productivo y coordinar capacidades para evaluar el potencial de protección de los resultados de investigación en la perspectiva de generar una fuente de ingresos que pueda provenir ya sea por la explotación exclusiva mediante un uso directo del objeto protegido, o bien, a través de terceros mediante el otorgamiento de licencias de explotación a cambio de regalías o royalties, la explotación en forma conjunta con terceros, o la venta de patentes.

Para la operación de estas unidades organizacionales se debe contar con el apoyo de sistemas de información junto con el de plataformas tecnológicas de última generación de soporte conducentes a apoyar la gestión de los DPI. Estos sistemas deben contener, entre otros, información relativa a patentes e información tecnológica, jurisprudencia, estadísticas de propiedad intelectual, herramientas de clasificación.

Lo expuesto ha dado origen a la Red de Propiedad Intelectual en Latinoamérica (proyecto PILA), conformada inicialmente por 18 universidades latinoamericanas y 4 universidades europeas. Este proyecto tiene por objetivo crear una plataforma de aprendizaje para intercambiar prácticas de gestión de propiedad intelectual $(\mathrm{PI})$ en las instituciones de educación superior, con el propósito de incentivar la interacción entre las universidades y las empresas, contribuyendo con ello al desarrollo económico y social de los países (PILA, 2009).
Se asume que una apropiada gestión de los DPI en las universidades alienta la innovación a través de la comercialización de los resultados de la investigación que realizan en sus laboratorios con beneficios en términos de ingresos por ventas o licenciamientos de patentes, colaboraciones con empresas, apoyos a consultorías y contratos de investigación, creación de parques tecnológicos e incubadoras de empresas, etc.

Utilizando la metodología AIDA (Jones, 1992) para evaluar las prácticas de PI sobre 120 universidades de 18 países latinoamericanas, los resultados señalan que si bien se ha avanzado en la creación de unidades u oficinas de PI y TT, queda mucho que recorrer en el ámbito de la gestión de los DPI vinculados a los proyectos de investigación que se llevan a cabo en las universidades. Esta metodología asume que una apropiada explotación de la PI se desarrolla en los siguientes niveles sucesivos: Atención (A), nivel asociado al conocimiento de los aspectos relacionados con la PI; Interés (I), nivel asociado a la protección de la PI de forma más o menos regular y sistemática; Deseo (D), nivel asociado a la gestión de los derechos de su PI; y Acción (A), nivel asociado a la explotación de sus derechos de su PI.

En Chile, esta metodología fue aplicada sobre II universidades, revelándose que (PILA, 2009):

- Mientras unas universidades muestran altos niveles de concienciación sobre la protección de información confidencial, en otras no existen directrices en cuanto a la protección de datos;

- Existe una ausencia o baja consolidación de las políticas y unidades especializadas en PI;

- Existe un bajo nivel de desarrollo en la gestión de la PI, especialmente en lo que concierne a la gestión operativa;

- Existe un bajo nivel de explotación de la PI por ausencia de estrategias institucionales en materia de PI y de políticas de defensa de la PI.

En la figura I se puede observar que en este plano, las universidades entrevistadas se encuentran en una fase intermedia y que se deben dar pasos orientados a la gestión y la explotación de la PI. 


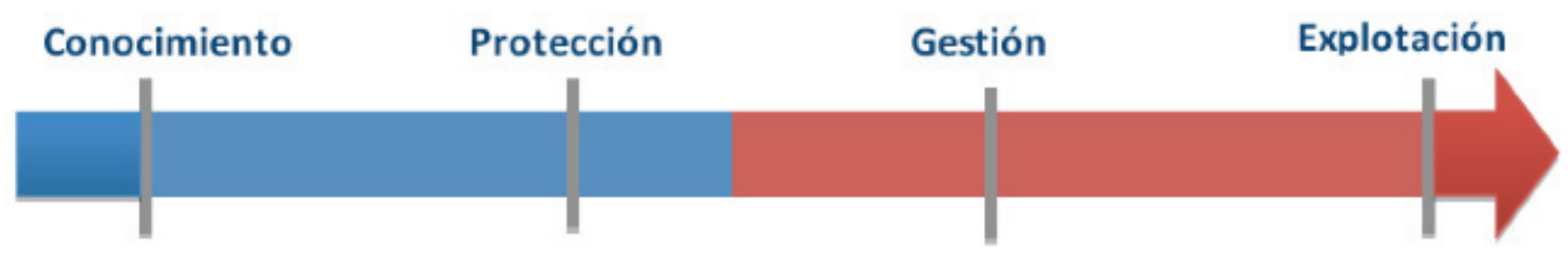

Figura I: Las fases de desarrollo según la metodología AIDA (PILA, 2009)

\section{El caso de la Universidad de Talca}

La Universidad de Talca (Klein, 2008), integrada a la red PILA, no escapa a la realidad descrita, razón por la cual, dentro de su modelo de negocio asociado a la comercialización y explotación de los DPI resolvió incrementar su vinculación con el medio y alentar la investigación aplicada por la vía de fortalecer los DPI sobre los resultados que emerjan de sus proyectos de investigación. Esta decisión está respaldada por la existencia en la Universidad de 7 Centros Tecnológicos ${ }^{4}$ destinados a contribuir al desarrollo empresarial en la región, en el país y el mundo. Estos centros constituyen el principal vínculo directo que posee la Universidad para relacionarse con la comunidad mediante convenios y proyectos de investigación y desarrollo, respaldados por la infraestructura y los conocimientos de académicos, especialistas y alumnos (UTalca, 2010).

La primera acción explícita conducente a disponer de una política de gestión de la propiedad intelectual, fue desarrollada en el año 2006, y estuvo constituida por la decisión de generar un estatuto de DPI, el cual fue modificado el año 2008 (UTalca, 2006; UTalca, 2008). Este estatuto tiene por objeto regular e incentivar los DPI en la Universidad, y en él se definió el modelo de distribución de utilidades en los casos que la Universidad licencie, transfiera o explote comercialmente su propiedad intelectual. Este modelo establece la participación económica en los beneficios netos de la comercialización o licenciamiento de las patentes a los autores, inventores o diseñadores que hayan contribuido en el desarrollo u obtención del objeto de la patente en su condición de académicos de la
Universidad. La distribución de los beneficios netos está fijada en un tercio para el(los) académicos y colaboradores directos del objeto de la patente, un tercio para la unidad de la Universidad (Facultad, Instituto o Laboratorio) en que se haya desarrollado la investigación que dio origen a la propiedad intelectual, y un tercio para la Universidad.

Para implementar la política de gestión de los DPI contenida en el estatuto, en el año 2008 se estableció una Dirección de Transferencia y Centros Tecnológicos (DTCT) dependiente de la Vicerrectoría Académica. Durante el año 2010, la Universidad modifica su estructura y crea la Vicerrectoría de Innovación, Desarrollo y Transferencia Tecnológica, incorporando dentro de sus unidades a la Dirección de Transferencia Tecnológica y la Unidad de Patentamiento, las cuales abordan el trabajo realizado hasta entonces por la DTCT.

Entre los propósitos con que fue creada esta Dirección destacan: difundir la información de libre disponibilidad existente en torno a los DPI al interior de la universidad; y facilitar el seguimiento administrativo de los procesos asociados a los DPI. Para el cumplimiento de estos propósitos se le asignaron las siguientes funciones: I) recibir y registrar las notificaciones de invenciones de académicos o administrativos de la Universidad; 2) gestionar la protección intelectual de resultados de investigación; y 3) crear los mecanismos que permitan el aprovechamiento de los resultados de investigación y que puedan ser transferidos a los sectores social y productivo.

\footnotetext{
${ }^{4}$ Estos Centros son: de Geomática, de Investigación y Transferencia en Riego y Agroclimatología, de la Industria de la Madera, de la Vid y el Vino, de Suelos y Cultivos, del Álamo, y de Pomáceas.
} 


\section{Desarrollo}

A continuación se presenta el desarrollo de una plataforma web para la gestión de los procesos de negocio vinculados a los DPI de los resultados de proyectos de investigación que se llevan a cabo en la Universidad de Talca y que apunta a contribuir al logro de los objetivos de la Dirección de Transferencia Tecnológica y la Unidad de Patentamiento.

En una primera etapa se procedió a conocer la realidad de la PI en las universidades chilenas; luego se definió un modelo de procesos vinculados a los DPI; y finalmente se desarrolló una plataforma web orientada a facilitar la gestión de las actividades asociadas a los DPI sobre la base del modelo de procesos definido.

\section{Etapa I: Investigación}

La fase de investigación fue realizada mediante una exploración bibliográfica (Gauna, 2006; Macho-Stadler et al., 2007; McAdam et al., 200I; Nelson, 2004; Rasmussen et al., 2006; Siegel et al., 2004; Thursby et al., 2009), exploración en sitios web de universidades, y reuniones en terreno con los participantes y responsables de los DPI en la Universidad de Talca y en la Universidad de Concepción por tratarse esta última de la universidad chilena que se encuentra a la vanguardia en estas materias.

\section{Etapa 2: Definición del Modelo de Procesos}

Para la definición y establecimiento del modelo de procesos se aplicó la notación BPMN (Business Process Modeling Notation) (White, 2008; Havey, 2005) destinada a la representación de procesos y considerando como base un primer modelo construido por Schmal y otros (20I0). La herramienta de software empleada fue Bizagi por su facilidad de uso (Bizagi, 2010). El proceso de definición del modelo se realizó con directa participación de sus principales actores, quienes describieron la realidad actual y sugirieron las mejoras a efectuar. Dicho modelo plasma la lógica de procesos, roles, actividades, tareas, estados y reglas de negocio que interactúan a lo largo del proceso.

\section{Etapa 3: Desarrollo de la Plataforma}

Para el desarrollo de la plataforma se aplicó la metodología de desarrolloágil de proyectos XP (eXtreme Programming) (Beck, 2004) dado que permite un rápido avance y validación de los requerimientos planteados al trabajarse en estrecha relación con sus usuarios para el desarrollo. Su limitación reside en la alta dependencia respecto de los actores claves, ya que sin la participación activa de ellos, el proyecto puede retrasarse considerablemente o simplemente llegar a su no cumplimiento.

\section{Resultados}

\section{El Modelo De Procesos}

La construcción del modelo se realizó sobre la base del levantamiento de requerimientos resultante de la investigación realizada y que se traduce en el modelo de los procesos identificados bajo los estándares seguidos por INAPI y consideraciones propias de los reglamentos relacionados a los Derechos de Propiedad Industrial.

El modelo de procesos resultante, graficado en la figura 2 , consta de un macroproceso, destinado a representar el esquema general de los procesos que deben ser llevados a cabo para gestionar una solicitud de patente, el cual se identifica como el objeto de negocio, cuyo ciclo de vida se inicia con el reporte de invención inicial hasta llegar a convertirse en una solicitud de patente, y posteriormente, dependiendo de los resultados, en una patente concedida. Este macroproceso está constituido por los siguientes 6 procesos:

a) Evaluación Externa: realizado por terceros, su objetivo es determinar la viabilidad de la patentación de la invención. En este análisis se deben determinar tres aspectos claves para la patentación: no obviedad, novedad y aplicabilidad. De cumplirse estos requisitos, se procede al proceso siguiente de evaluación interna.

b) Evaluación Interna: destinada a determinar el interés de la universidad por la patentabilidad sobre la invención. En caso de no interesarse, la universidad deja en libertad de acción al investigador, desligándose de la propiedad de la invención y liberando al investigador para la búsqueda de financiamiento ante terceros para su patentación. La confección del acta oficial es realizada por el Comité de Propiedad Industrial de la Universidad de Talca. 
c) Titularización de Invención: tiene por fin realizar todas las gestiones que dicen relación con la postulación de la invención y la confección de la solicitud para su patentación en INAPI. La Universidad de Talca tiene un rol activo en este proceso, recopilando la información y documentación necesaria para la confección de la solicitud de patente. El resultado final de este proceso concluye con la presentación de la solicitud de patente en INAPI.

d) Proceso de Pagos: se refiere al pago de compromisos legales que deben ser cancelados por conceptos de pagos de derechos, tasas, compromisos con terceros y otros pagos relacionados con las solicitudes de patentes. La realización de los pagos correspondientes autoriza a seguir avanzando en las etapas del proceso.

e) Comercialización de Patentes: tiene por objeto la obtención de beneficios económicos, dentro del periodo de exclusividad, por la patente concedida. Estos beneficios pueden ser obtenidos mediante diversos tipos de acuerdos con terceros, ya sean franquicias, licencias u otros. La obtención de recursos es fundamental, para cubrir los costos de investigación y gestión en los que se ha incurrido, además de posibilitar la investigación y desarrollo de nuevas invenciones.

g) Explotación de patentes: su objetivo es recepcionar los pagos que efectúan terceros a la universidad al haber adquirido compromisos por conceptos de franquicias, licencias u otros, por el uso de las invenciones patentadas pertenecientes a la Universidad de Talca. 


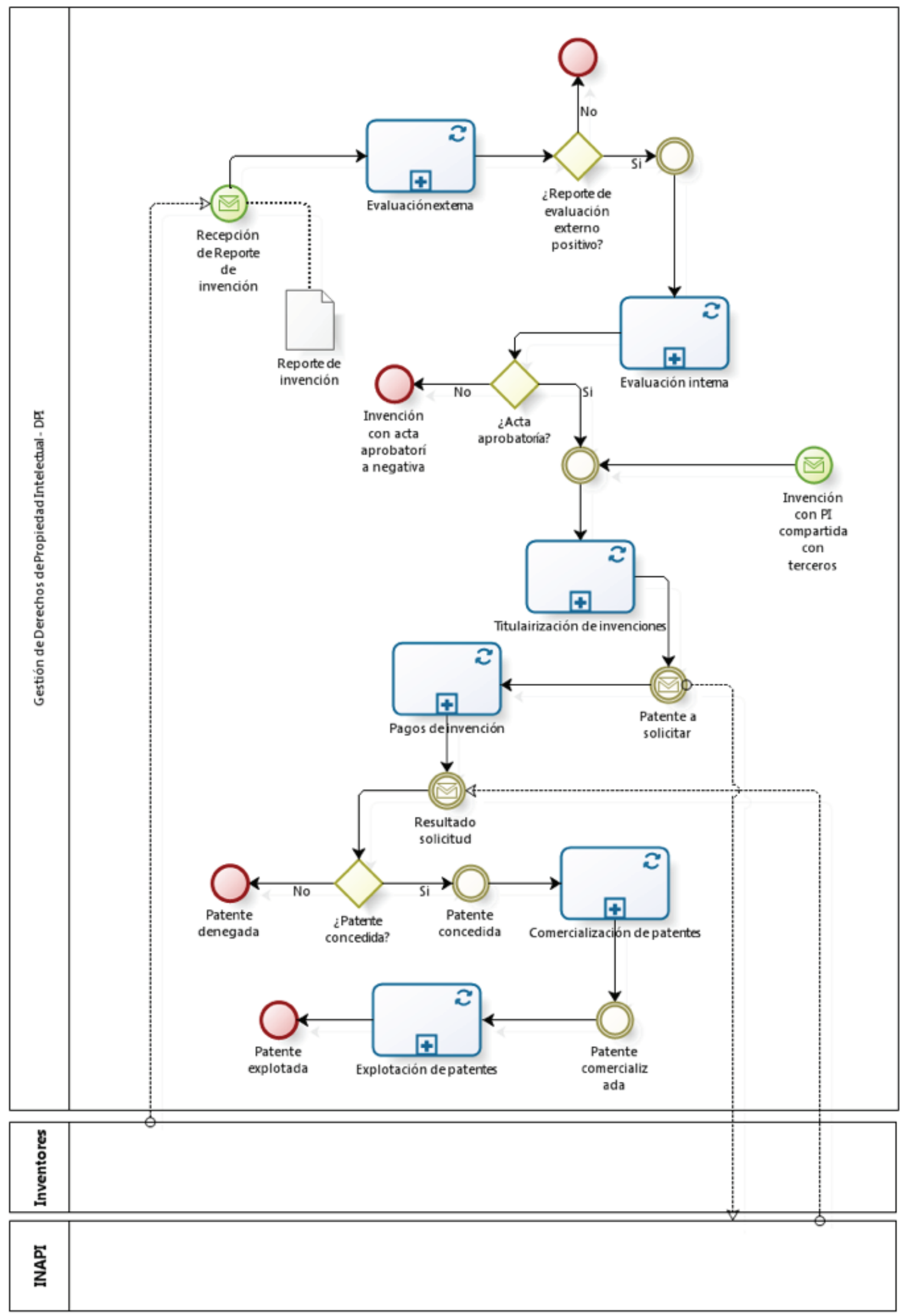

Figura 2. Modelo de Procesos Vinculado a los DPI./ Fuente: Elaboración propia 
Estos procesos dieron origen a los siguientes roles, tareas, estados, decisiones de negocio y documentos:

a) Roles: Unidad de Patentamiento, Consultor, Comité de Propiedad Intelectual, Secretaria Técnica, Abogado, Titularizador, Área Técnica, Área Legal, Inventor, Propiedad Industrial Terceros, Dirección Jurídica Utalca, Cliente, Vendedor y Trazador;

b) Estados del macroproceso: Recepción de Reporte de Invención, Invención con evaluación externa positiva, Invención con evaluación externa negativa, Invención a apropiar por la Universidad de Talca, Invención con acta aprobatoria negativa, Invención con PI compartida con terceros, Patente a solicitar, Resultado solicitud, Patente concedida, Patente denegada, Patente comercializada, y Patente explotada;

c) Decisiones: ¿Reporte de evaluación externo positivo? ¿Acta aprobatoria? ¿Patente concedida? ¿No obvio? ¿Novedoso? ¿Aplicable? ¿Involucra Propiedad Industrial de terceros?;

d) Documentos: Reporte de invención, Reporte de evaluación externa, Acta comitéPI, Solicitud de información para titularización de la invención, Documentación solicitada para titularización de la invención, Formulario solicitud de patente, Estatutos de PI de la Universidad de Talca, Documento tipo para cesión de derechos, Acuerdo preliminar con terceros, Condiciones de acuerdo, Formulario solicitud de patente, y Comprobante ingreso formulario de solicitud de patente.

\section{Plataforma Web Para La Gestión De Patentes}

El desarrollo de la Plataforma Web fue abordado mediante el uso de tecnología Asp.Net, utilizando el entorno de desarrollo de Visual Studio.Net bajo el lenguaje Visual Basic.net. Para la base de datos del sistema se utilizó SQL Server Express Edition. Los usuarios identificados fueron los visitantes, definidos como el público en general, los investigadores, los administradores de proyectos y los administradores de patentes, cuyas funciones se presentan en la Tabla 3.

En las figuras que siguen se muestra el mapa con que fue concebido el sitio de la plataforma web (figura 3); la interfase constituida por un front-office para la interacción con los usuarios no registrados (figura 4) y los tres módulos para los usuarios registrados en la plataforma: Investigadores, Administradores de Proyectos y Administradores de Patentes (figuras 5,6 y 7 ).

\begin{tabular}{|l|l|l|}
\hline \multicolumn{1}{|c|}{ Usuarios } & \multicolumn{1}{|c|}{ Función } & Acceso \\
\hline Visitante & Consultar información de libre disponibilidad & $\begin{array}{l}\text { Secciones iniciales que no } \\
\text { requieran identificación }\end{array}$ \\
\hline Investigadores & $\begin{array}{l}\text { Enviar y revisar sus reportes de invención; revisar proyectos, } \\
\text { patentes y solicitudes de patentes; descargar documentos }\end{array}$ & Módulo de Investigadores \\
\hline $\begin{array}{l}\text { Administrador de } \\
\text { Proyectos }\end{array}$ & Registrar investigadores y proyectos & $\begin{array}{l}\text { Módulo de Administración de } \\
\text { Proyectos }\end{array}$ \\
\hline $\begin{array}{l}\text { Administrador de } \\
\text { Patentes }\end{array}$ & $\begin{array}{l}\text { Generar y resolver reportes de invención; gestionar patentes, } \\
\text { solicitudes de patentes, contratos y ventas }\end{array}$ & $\begin{array}{l}\text { Módulo de Administración de } \\
\text { Patentes }\end{array}$ \\
\hline $\begin{array}{l}\text { Administrador } \\
\text { Global }\end{array}$ & Administrar el sistema, registrar usuarios y publicar contenidos & A todo el sistema \\
\hline
\end{tabular}

Tabla 3: Usuarios y sus funciones y accesos 


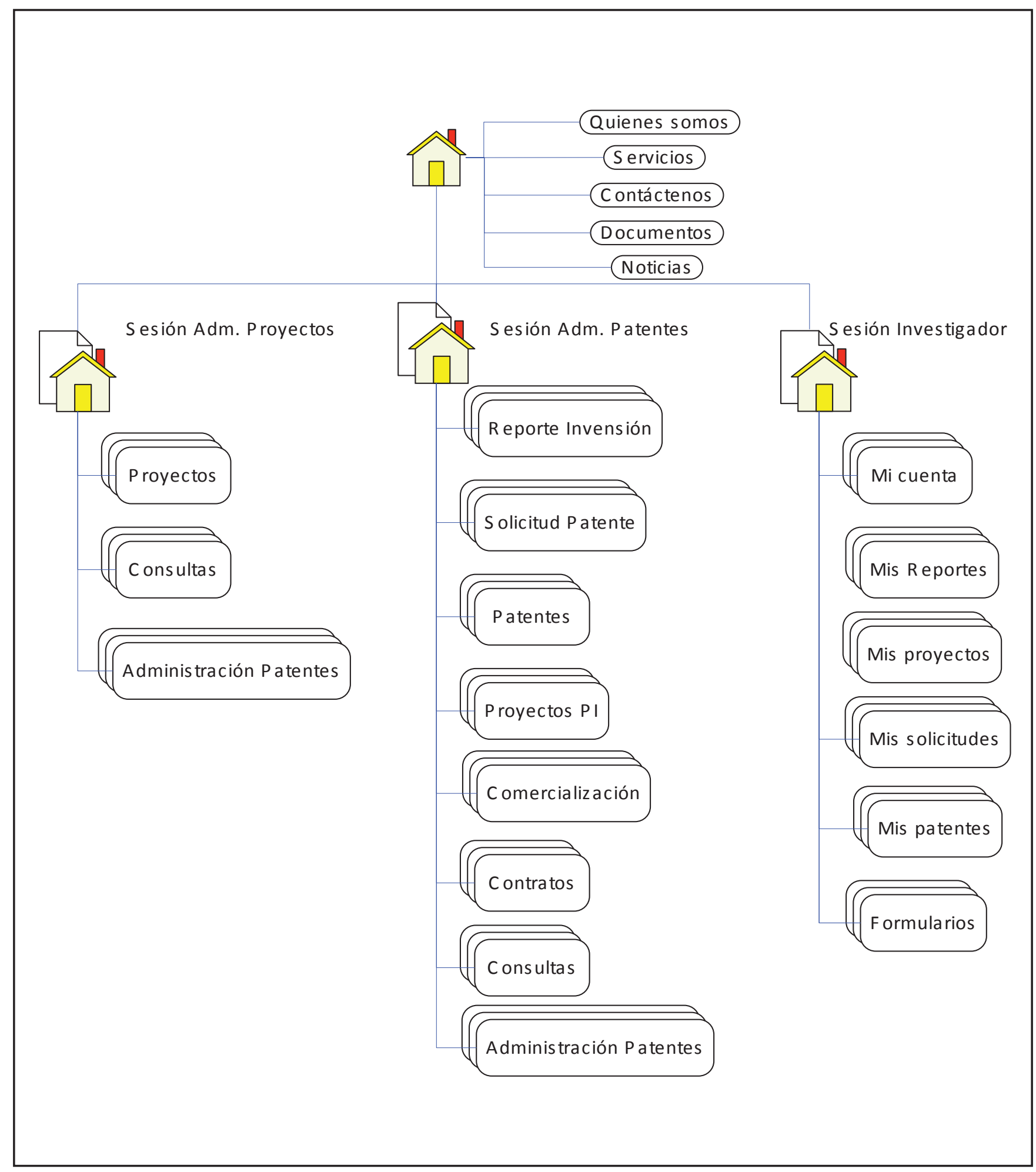

Figura 3. Mapa de Navegación 


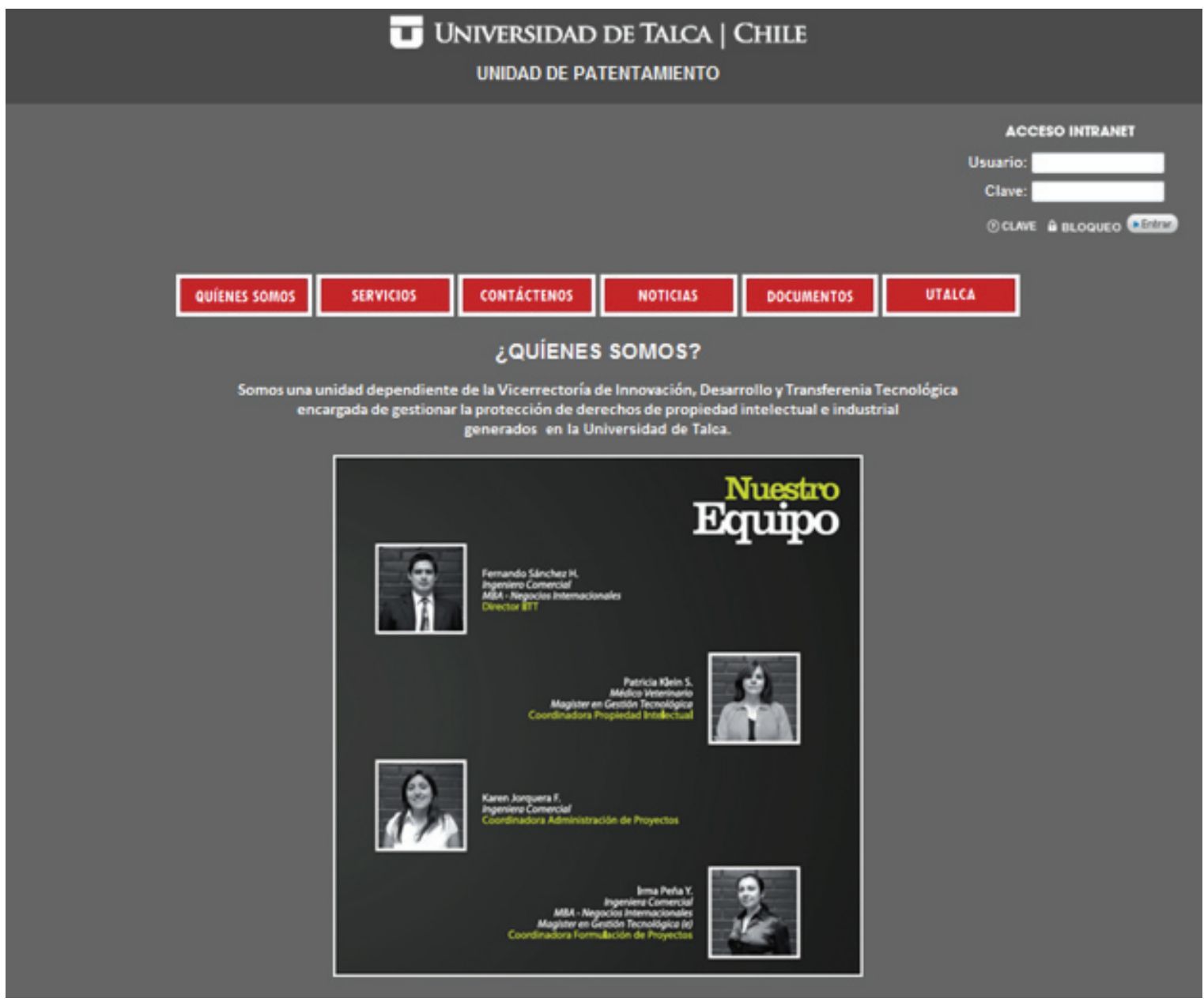

Figura 4. Interfase de inicio

\section{MÓDULO INVESTIGADORES}

\section{Diego Acuña Bastías (salir)}

Inicio Proyectos Reportes Invención Patentes Documentos Mi Cuenta
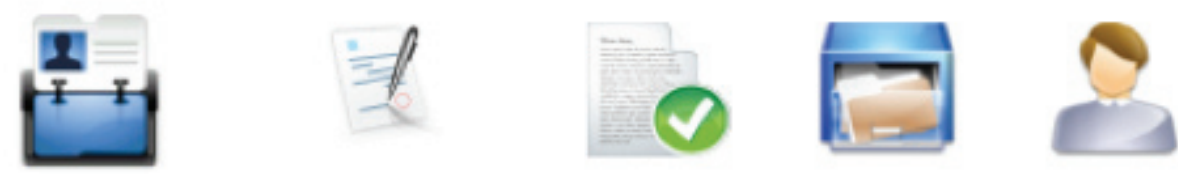

Mis Proyectos $\underline{\text { Reporte Invención Mis Patentes Documentos Mi Cuenta }}$

Figura 5. Interfase Sesión Investigadores 


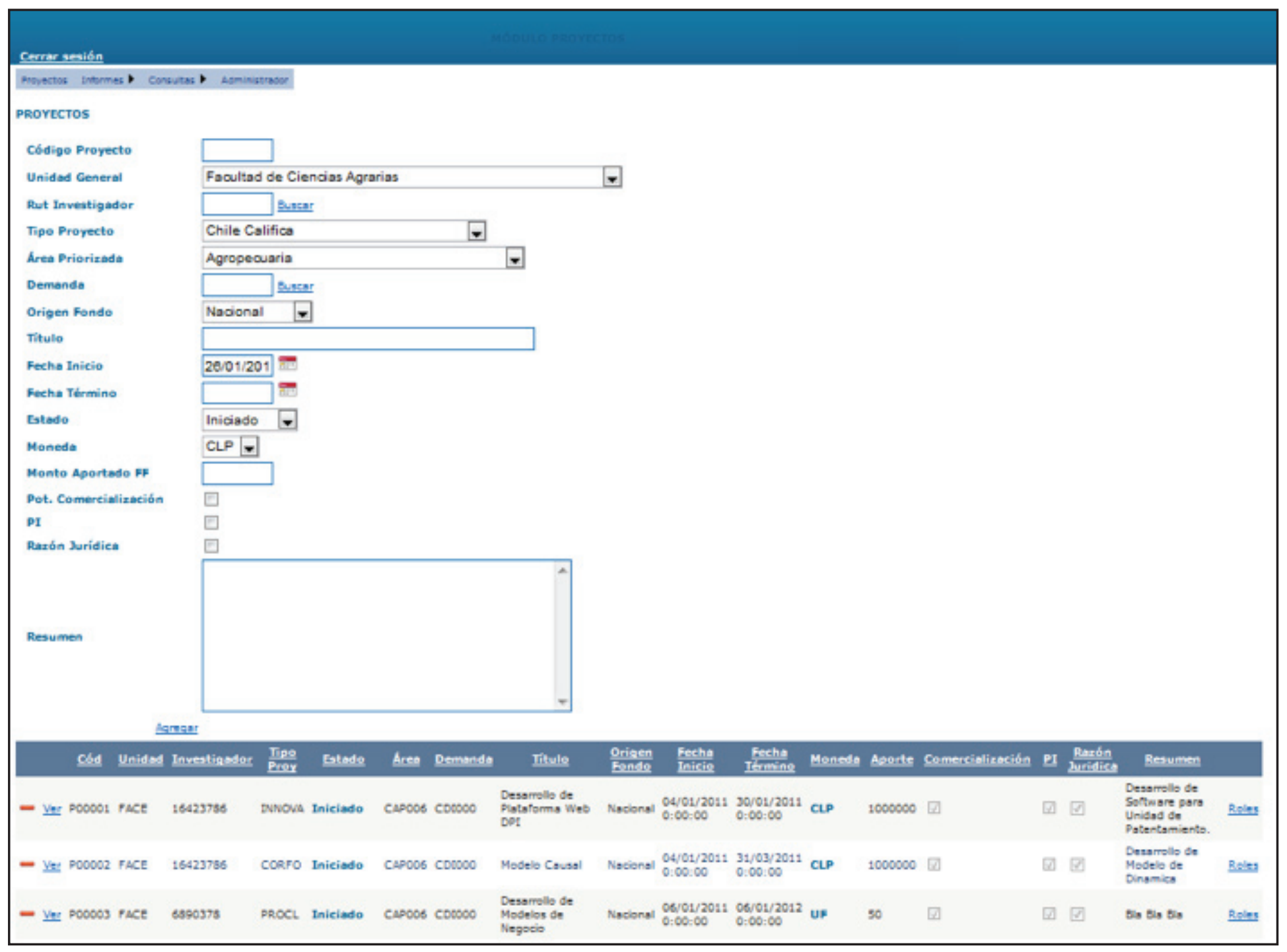

Figura 6. Interfase Sesión Administración de Proyectos 
Cerrorsesión

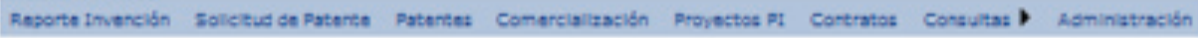

Reportes activos

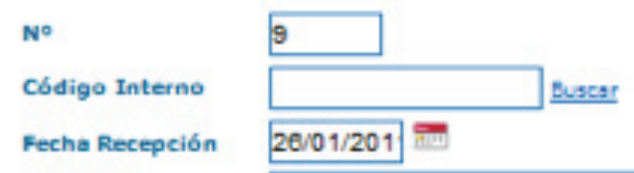

Solicitante

Titule

Beneficio Terceros

Aarsaar Caneslar

\begin{tabular}{|c|c|c|c|c|c|c|c|c|c|}
\hline No Reporte & & Q Estado Reporte & \begin{tabular}{|l|l} 
No Evaluado & $\mathbf{Q}$
\end{tabular} & & & & & & \\
\hline № & Prexideste & Beschas & Iftule & Solicitante & Tercetros & & Eatade & Archive & \\
\hline - Ver 1 & P00001 & $\begin{array}{l}05 / 01 / 2011 \\
0: 00: 00\end{array}$ & Dessrrollo de Plataforma Web DPI & $\begin{array}{l}\text { Diege Aeuns, } \\
\text { Bastioss }\end{array}$ & ㅁ] & Iersenses & Soliotado & Anching & $\frac{\text { Carrar }}{\text { Archive }}$ \\
\hline- Ver 2 & 900002 & $\begin{array}{l}05 / 01 / 2011 \\
0: 00: 00\end{array}$ & Modela Caussl & $\begin{array}{l}\text { Diego Acung } \\
\text { Bastiss }\end{array}$ & $\square$ & Terceress & Soliotede & Aechive & $\begin{array}{l}\text { Gorser } \\
\text { Archive }\end{array}$ \\
\hline$-\operatorname{Ver}^{3}$ & 900003 & $\begin{array}{l}06 / 01 / 2011 \\
0: 00: 00\end{array}$ & Destarrollo de Modelos de Negocio & Rodelfo Sehmal & $\nabla$ & $\underline{\text { Terseres }}$ & Soliotade & Aechive & $\begin{array}{l}\text { Carear } \\
\text { Arshive }\end{array}$ \\
\hline - Ver 4 & 900004 & $\begin{array}{l}20 / 12 / 1999 \\
0: 00: 00\end{array}$ & 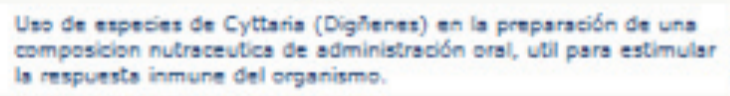 & $\begin{array}{l}\text { Guillerme } \\
\text { Schmeda } \\
\text { Hirselmann }\end{array}$ & $\square$ & Terceress & Soliotedo & Aechive & $\begin{array}{l}\text { Sorsor } \\
\text { Arehive }\end{array}$ \\
\hline$-\operatorname{Ver} 5$ & 900005 & $\begin{array}{l}12 / 01 / 2011 \\
0: 00: 00\end{array}$ & Anslisis en productos de minering & $\begin{array}{l}\text { Ense Ramos } \\
\text { Arellono }\end{array}$ & $\square$ & Terseress & $\begin{array}{l}\text { Evaluado } \\
\text { Externo }\end{array}$ & Aechive & $\frac{\text { Cargar }}{\text { Archive }}$ \\
\hline - yer 6 & 900006 & $\begin{array}{l}12 / 01 / 2011 \\
0.00: 00\end{array}$ & Proyecto de Inpenieris 1 & $\begin{array}{l}\text { Felipe Flores } \\
\text { Leyton }\end{array}$ & $\square$ & Iterceros & $\begin{array}{l}\text { Evalusdo } \\
\text { Interno }\end{array}$ & Archivo & $\begin{array}{l}\text { Correr } \\
\text { Archive }\end{array}$ \\
\hline- Ver 7 & 900007 & $\begin{array}{l}12 / 01 / 2011 \\
0: 00: 00\end{array}$ & Desarrollo de productes para mejora de siatemas de riego & $\begin{array}{l}\text { Rodrigo Palomo } \\
\text { Vninz }\end{array}$ & $\square$ & Terseres & $\begin{array}{l}\text { No } \\
\text { Evaluado }\end{array}$ & Aechive & $\begin{array}{l}\text { Corger } \\
\text { Arehive }\end{array}$ \\
\hline$-\underline{\operatorname{Vnr}} s$ & 900000 & $\begin{array}{l}12 / 01 / 2011 \\
0: 00: 00\end{array}$ & Desurrolo Plataforms Wab Gertion Propindad Industrial & $\begin{array}{l}\text { Diego Aculs } \\
\text { Bastios }\end{array}$ & $\square$ & Terenrar & Solietnde & Anething & $\frac{\text { Cargar }}{\text { Arshive }}$ \\
\hline
\end{tabular}

Figura 7. Interfase Sesión Administración de Patentes 


\section{Conclusiones}

La protección de los DPI en las universidades chilenas se encuentra en su fase inicial, solo con casos aislados de "éxito" destacándose el de la Universidad de Concepción, universidad regional que concentra del orden del $30 \%$ del total de patentes concedidas a las universidades, siendo una importante referencia para las demás universidades interesadas en incursionar en este ámbito. Tres son los factores clave que lo explican: I) Cuentan con un calificado equipo de profesionales vinculados a las principales áreas de desarrollo de patentamiento de la universidad, donde cuyos roles, tareas y responsabilidades están especificados claramente; 2) Todos los procesos implicados en la gestión de $\mathrm{PI}$ son realizados internamente, $\mathrm{y}$ dado el alto volumen de transacciones que manejan, les permite operar con un bajo costo por gestión de patentes; y 3) Poseen una consolidada relación con el medio, reflejado en una alta interacción con las empresas regionales.

Si bien actualmente no cuentan con un sistema de gestión formalmente establecido, tienen claro conocimiento de los aportes que este tipo de herramientas otorga a la gestión de las organizaciones, razón por la que se encuentran analizando la construcción de un sistema que facilite la gestión en $\mathrm{PI}$.

Para avanzar significativamente en estas materias es fundamental que se consideren aspectos como la definición de claros procesos que permitan entregar las bases para la gestión, establecer equipos de trabajo, así como establecer fuertes relaciones con el medio para el logro de alianzas que faciliten el éxito, además de identificar los factores de desempeño que serán evaluados en cada una de las etapas para medir el éxito de la gestión realizada, apoyándose de herramientas que permitan monitorear y controlar la gestión de las actividades del negocio mediante indicadores claves.

Si bien la concesión de una patente, ya es una buena señal, no es el fin último. Para evaluar su éxito es fundamental lograr la transferencia tecnológica hacia el mercado. De lo contrario se está solo en potencia de buenos resultados de gestión, pero no de buenos resultados de aplicabilidad.

El sistema construido sobre una plataforma web representa el soporte tecnológico que permite estandarizar y agilizar el proceso de negocios para la gestión de patentes en la Universidad de Talca al establecer los procesos de manera lógica y controlada, donde están identificados sus actores, los cuales tienen roles, privilegios y accesos particulares, dependiendo de las actividades que cada una de ellos debe realizar. Además permite identificar el ciclo de vida del objeto de negocios especificando claramente en qué estado se encuentra cada uno de ellos.

Además del seguimiento particular a cada una de las invenciones que posteriormente pasan dan origen a solicitudes y eventualmente patentes, el sistema permite: a) manejar información consolidada sobre la gestión del proceso de patentes; b) desplegar informes y reportes que serán fundamentales para las mejoras propias del proceso; y c) apoyar la toma de decisiones en los casos que sea requerido.

En la actualidad, luego de haber sido construido y probado satisfactoriamente, el sistema se encuentra en la fase de puesta en marcha para su operación plena en el año 2012.

De los antecedentes señalados se debe destacar que el sistema en sí mismo solo tiene valor si está inserto en una universidad con una clara vocación y capacidad de investigación y de orientación hacia su entorno, en cuyo caso el sistema se constituye en un puente que integra la actividad de investigación y de vinculación con el medio. 


\section{Referencias}

BECK K. Andres C. (2004). Extreme Programming Explained. Ed. Addison-Wesley, USA. 224 pp.

BIZAGI DOCUMENTATION CENTER. (20I0). BizAgi, Guía de referencia BPMN. http://wiki.bizagi.com/es [consultado el 20 Octubre 2010].

BRUNNER, J.J. (2009). Educación Superior en Chile: Instituciones, Mercados y Políticas Gubernamentales (19672007). Ed. Universidad Diego Portales. Santiago-Chile.

GAUNA A. Nesta L. (2006). University patenting and its effects on academia research: The emerging European evidence. Research Policy, 35, 795-807.

GIBBONS, M. Limoges, C. Schwartzman S. Scott P. Trow, M. (2004). The new production of knowledge: the dynamics of science and research in contemporary societies. SAGE Publications, Londres-Inglaterra.

HAVEY M. (2005). Essential Business Process Modeling. Ed. O'Reilly, USA

INSTITUTO NACIONAL DE PROPIEDAD INDUSTRIAL. (20I0). Bases de Datos INAPI. http://ion.inapi.cl [consultado el 5 de Octubre 20I0].

JONES, J. C. (1992). Design methods, Ed. John Wiley \& Sons, New York-USA.

KLEIN P. (2008). Transferencia de resultados de investigación en universidades: revisión, análisis y propuestas. Proyecto final para optar al grado de Magister en Gestión Tecnológica con énfasis en biotecnología.

MACHO-STADLER I. Pérez-Castillo D. Vengelus R. (2007). Licensing of university inventions: the role of a technology transfer office. International Journal of Industrial Organization, 25, 483-510.

McADAM R. Keogh W. Galbraith B. Laurie D. (2005). Defining and improving technology transfer business and management processes in university innovation centers. Technovation, 25, 1418-29.
MOVERY D. Nelson R. Sampat B. Ziedonis A. (200I). The growth of patenting and licensing by US universities: an assessment of the effects of the Bayh Dole act of 1980. Research Policy, 30, 99-119.

NELSON R. (2004). The market economy and the scientific commons. Research Policy, 33(3), 455-7I.

OMPI (2009). Indicadores Mundiales de Propiedad Intelectual de la Organización Mundial de Propiedad Intelectual. http://www.wipo.int/ipstats/es/statistics/patents/ pdf/94IS 2009.pdf [consultado el I3 de Octubre 2010]

PILA: Red de Propiedad Intelectual e Industrial en América Latina. (2009). Gestión de Propiedad Intelectual e Industrial en Instituciones de Educación Superior. http://www. pila-network.org/ [consultado el 13 de Octubre 2010].

PLÁCIDO J. Vitale A. (2009). As patentes nas universidades: análise dos depósitos das universidades públicas paulistas (1995-2006). Ci. Inf., 38(2), 9-I8.

RASMUSSEN E. Moen O. Gulbrandsen M. (2006). Initiatives to promote commercialization of university knowledge. Technovation, 26, 518-33.

SAMPAT B. Movery D. Ziedonis A. (2003). Changes in university patent quality after the Bayh Dole Act: A reexamination. International Journal of Industrial Organization, 2I, I37I-90.

SCHMAL R. López S. Cabrales F. (2006). El camino hacia la patentación en las universidades. Ingeniare. Revista Chilena de Ingeniería, 13(3), 172-86

SCHMAL R. López S. Cabrales F. Acuña, D. (2010). Modelado de Procesos de Negocio para la Gestión de patentes en Universidades. Información Tecnológica, 2I(6), 67-78.

SIEGEL D. Waldman D. Leanne A. Link A. (2004). Toward a model of the effective transfer of scientific knowledge from academicians to practicioners: quality evidence from the commercialization of university technologies. Journal of Engimeering and Technology Management, 2I(I-2), II5-42.

THURSBY J. Fuller A. Thursby M. (2009). US faculty patenting: inside and outside university. Research Policy, 38, 14-25. 
U. DE TALCA. (2010). Centros Tecnológicos. http://www. utalca.cl/link.cgi//CentrosTecnologicos/ [consultado el 15 de Diciembre 2010].

WHITE S.A. Miers D. (2008). BPMN Modeling and reference guide. Future Strategies Inc. Home, USA.

ZALDÍVAR A. Oconnor L. (2010). Una experiencia cubana en la gestión por la innovación desde las patentes universitarias. Revista Universitaria de Investigación y Diálogo Académico, 6(I), 96-124. http://conhisremi.iuttol.edu.ve/ pdf/ARTI000090.pdf [consultado el 10 de octubre 20II] 
Anexo. Ranking de universidades chilenas según número de patentes solicitadas y concedidas (197I-20I0)

\begin{tabular}{|c|c|c|c|}
\hline $\mathbf{N}^{\mathbf{o}}$ & Institución & Solicitadas & Concedidas \\
\hline 1 & Universidad de Concepción & 101 & 36 \\
\hline 2 & Universidad Técnica Federico Santa María & 50 & 10 \\
\hline 3 & Universidad de Chile & 50 & 16 \\
\hline 4 & Universidad Católica de Chile & 39 & 12 \\
\hline 5 & Universidad de Santiago & 37 & 11 \\
\hline 6 & Universidad Católica de Valparaíso & 20 & 3 \\
\hline 7 & Universidad Austral & 14 & 2 \\
\hline 8 & Universidad Católica del Norte & 10 & 2 \\
\hline 9 & Universidad de Antofagasta & 9 & 0 \\
\hline 10 & Universidad de la Frontera & 7 & 0 \\
\hline 11 & Universidad Arturo Prat & 6 & 0 \\
\hline 12 & Universidad Católica de Temuco & 5 & 0 \\
\hline 13 & Universidad del Bio Bio & 5 & 0 \\
\hline 14 & Universidad de los Lagos & 4 & 1 \\
\hline 15 & Universidad Tecnológica Metropolitana & 4 & 0 \\
\hline 16 & Universidad de Talca & 4 & 1 \\
\hline 17 & Universidad Católica de la Santísima Concepción & 3 & 0 \\
\hline 18 & Universidad de Valparaíso & 3 & 1 \\
\hline 19 & Universidad de la Serena & 2 & 2 \\
\hline 20 & Universidad de Magallanes & 1 & 1 \\
\hline 21 & Universidad Católica del Maule & 0 & 0 \\
\hline 22 & Universidad de Playa Ancha de Ciencias de la Educación & 0 & 0 \\
\hline 23 & Universidad Metropolitana de Ciencias de la Educación & 0 & 0 \\
\hline 24 & Universidad de Tarapacá & 0 & 0 \\
\hline \multirow[t]{2}{*}{25} & Universidad de Atacama & 0 & 0 \\
\hline & Totales & 374 & 98 \\
\hline
\end{tabular}

Fuente: Elaboración propia a partir de datos disponibles en INAPI (http://ion.inapi.cl) 
J. Technol. Manag. Innov. 20II,Volume 6, Issue 4 